\title{
Correlation between KIT expression and KIT mutation in melanoma: a study of 173 cases with emphasis on the acral-lentiginous/ mucosal type
}

Carlos A Torres-Cabala ${ }^{1,2}$, Wei-Lien Wang ${ }^{1}$, Jonathan Trent $^{3}$, Dan Yang ${ }^{1}$, Su Chen ${ }^{1}$, John Galbincea ${ }^{1}$, Kevin B Kim ${ }^{3}$, Scott Woodman ${ }^{3}$, Michael Davies ${ }^{3}$, Jose A Plaza ${ }^{1}$, JW Nash ${ }^{1}$, Victor G Prieto ${ }^{1,2}$, Alexander J Lazar ${ }^{1,2}$ and Doina Ivan ${ }^{1,2}$

${ }^{1}$ Department of Pathology, The University of Texas-MD Anderson Cancer Center, Houston, TX, USA; ${ }^{2}$ Department of Dermatology, The University of Texas-MD Anderson Cancer Center, Houston, TX, USA and ${ }^{3}$ Department of Melanoma Medical Oncology, The University of Texas-MD Anderson Cancer Center, Houston, TX, USA

\begin{abstract}
The role of immunohistochemistry in the assessment of KIT status in melanomas, especially acral lentiginous/ mucosal, is not well established. Although the reported prevalence of $K I T$ mutations in acral lentiginous/ mucosal melanomas is relatively low, detection of mutations in KIT can have profound therapeutic implications. We evaluated the efficacy of immunohistochemistry to predict mutations in KIT. One hundred seventy-three tumors, comprising primary and metastatic melanomas (141 acral lentiginous/mucosal, 5 nodular, 4 lentigo maligna, 3 superficial spreading, 2 uveal, 1 melanoma of soft parts, 8 metastases from unclassified primaries, and 9 metastases from unknown primaries) were studied. Immunohistochemical expression of KIT using an anti-CD117 antibody and KIT mutational analysis by gene sequencing of exons 11, 13, and 17 were performed. Eighty-one percent of acral lentiginous/mucosal melanomas, primary and metastatic, showed KIT expression by at least $5 \%$ of the tumor cells. The overall frequency of activating $K I T$ gene mutations in acral lentiginous/ mucosal melanomas was $15 \%$ (14 out of 91 cases), being the L576P mutation in exon 11 the most frequently detected (4 of 14 cases). Cases showing less than $10 \%$ positive tumor cells were negative for KIT mutations. Eighty-two percent (12 of 14) of cases positive for KIT mutation showed KIT expression in more than $50 \%$ of the cells. An association between immunohistochemical expression of KIT and mutation status was found $(P=0.007)$. Immunohistochemical expression of KIT in less than $10 \%$ of the cells of the invasive component of acral lentiginous/mucosal melanomas appears to be a strong negative predictor of $K I T$ mutation and therefore can potentially be used to triage cases for additional KIT genotyping.
\end{abstract}

Modern Pathology (2009) 22, 1446-1456; doi:10.1038/modpathol.2009.116; published online 28 August 2009

Keywords: melanoma; acral lentiginous; mucosal; KIT; immunohistochemistry; mutation

The role of KIT signaling in melanocyte biology has been extensively studied. It was shown that the interaction of stem cell factor with KIT, its receptor, is critical for the survival, proliferation, differentiation and migration of melanocytes. ${ }^{1}$ However, the

Correspondence: Dr CA Torres-Cabala, MD, Departments of Pathology and Dermatology, The University of Texas-MD Anderson Cancer Center, 1515 Holcombe Blvd., Unit no. 85 , Houston, TX 77030-4009, USA.

E-mail: ctcabala@mdanderson.org

Received 2 June 2009; revised and accepted 27 July 2009; published online 28 August 2009 regulation of KIT pathway is complex and depends on multiple other cellular factors. ${ }^{2}$ Whereas KIT activation mutations are known to be associated with a variety of malignant human tumors such as gastrointestinal stromal tumor (GIST), seminoma, and mastocytosis/mast cell leukemia, ${ }^{3}$ the introduction of a KIT-activating mutation into an immortalized murine melanocyte cell line was reported to result in a motogenic more than a mitogenic effect. ${ }^{4}$ Therefore, it was speculated that to acquire proliferative advantage and escape from the epidermal boundaries, melanoma cells should lose KIT expression. ${ }^{4}$ This hypothesis was supported by previous 
observations in which KIT expression in melanoma was strong in the in situ and junctional component of invasive lesions, but was lost once the melanoma became invasive and metastatic. ${ }^{5,6}$

Certain types of invasive melanomas, however, were reported to express KIT. Most of the metastatic ocular melanomas were positive for KIT in a series of cases. ${ }^{7}$ Sporadic reports of rare metastatic melanomas harboring the L576P KIT-activating mutation and displaying strong and diffuse KIT expression ${ }^{8,9}$ suggested that in a subset of cases, melanoma progression would involve KIT activation rather than loss of KIT activity. An immediate theoretical use of tyrosine kinase inhibitors in these cases was proposed..$^{10}$ Although a trial using imatinib mesylate in melanomas without KIT mutations showed no overall benefit, use of tyrosine kinase inhibitors in cases with documented mutations has shown dramatic results. ${ }^{11-15}$ Subsequently, using array comparative genomic hybridization and mutation analysis, Curtin et $a l^{16}$ found that mucosal, acral, and sun-induced melanomas frequently show genetic amplification of the KIT locus and activating mutations of the gene. Although most of the mutation-positive cases showed elevated KIT protein expression, some of the analyzed tumors required higher antibody concentrations for the immunohistochemical detection of KIT. The fact that more than a third of the melanomas without detectable KIT mutation or copy number increase showed overexpression of KIT by immunohistochemistry led the authors to hypothesize that mechanisms other than gene mutation or amplification could explain the observed KIT overexpression. ${ }^{16}$ The role of immunohistochemistry in the assessment of KIT in acral lentiginous/mucosal melanomas and its relation to the mutational status of the KIT gene is, therefore, not well established.

The prevalence of KIT mutations in acral lentiginous/mucosal melanomas is relatively low (no more than $15-20 \%{ }^{16-19}$ ) but can have profound therapeutic implications for localized high risk or metastatic disease. In this study, we evaluate KIT protein expression by immunohistochemistry and KIT mutational status by PCR amplification and sequencing in a large series of melanomas with emphasis on acral lentiginous/mucosal melanomas. Our goal was to correlate the level of KIT expression and activating KIT gene mutations in these tumors to evaluate the efficacy of immunohistochemistry to discriminate potential candidates for targeted therapy.

\section{Materials and methods}

With appropriate Institutional Review Board approval, 177 tumors from 173 patients were selected from The University of Texas MD Anderson Cancer Center clinical database for the years 2005-2008. Primary tumors and their metastases were available for study in four patients. For purposes of statistical analysis, only one of the tumors (either primary or metastatic) was recorded from these four cases. The 173 analyzed melanomas comprised the following: 46 primary acral lentiginous, 24 metastatic acral lentiginous, 61 primary mucosal, 10 metastatic mucosal, three primary nodular, two metastatic nodular, two primary lentigo maligna, two metastatic lentigo maligna, three metastatic superficial spreading, two primary uveal, one melanoma of soft parts/clear cell sarcoma, eight metastatic melanomas from unclassified primaries, and nine metastatic melanomas from unknown primaries. Our series was enriched with a large number of acral lentiginous/mucosal melanomas as these tumors were the most likely to harbor mutations in KIT.

Glass slides and paraffin tissue blocks were retrieved from the Department of Pathology archives. All tissues had been fixed in neutralbuffered $10 \%$ formalin.

\section{Immunohistochemical Analysis}

Immunohistochemical analysis for KIT was performed using an anti-CD117 polyclonal rabbit antihuman antibody (Code A4502, dilution 1:100, Dako Cytomation, Carpinteria, CA, USA) on paraffin sections according to the BOND MAX protocol (Vision Biosystems, Norwell, MA, USA) guidelines. After incubation with Bond Dewax solution for $30 \mathrm{~min}$ at $72^{\circ} \mathrm{C}$, antigen retrieval was performed with citrate buffer for $15 \mathrm{~min}$ at $100^{\circ} \mathrm{C}$. Peroxidase block was done using a $3.0 \%$ hydrogen peroxide solution. The slides were then incubated with post-primary solution (polymer enhancer) for $8 \mathrm{~min}$. Polymer (poly-HRP anti-mouse/anti-rabbit IgG) was then applied on the glass slides for 8 min. The reaction was developed using DAB as chromogen and hematoxylin was used as counterstaining. Negative and positive controls were performed and examined in parallel.

Immunohistochemical-stained sections were evaluated for both tumor cell percentage and intensity of immunoreactivity. Percentage of positive cells was recorded as 0 (negative), $<5 \%$ of cells staining, $5-50 \%$ of cells staining, $51-95 \%$ of cells staining, and $>95 \%$ of cells staining. Intensity was scored as 0 (negative), $1+$ (weak), $2+$ (moderate), and $3+$ (strong).

\section{Mutational Analysis}

Mutational analysis was performed on 135 cases. In all, 106 of these were consecutive cases characterized in the Molecular Diagnostics Laboratory for clinical purposes and the remaining 29 cases were performed in a research laboratory at our institution.

Specific paraffin blocks containing tumor were identified and H\&E-stained and unstained $4 \mathrm{~mm}$ thick sections were obtained. Areas containing viable tumors were marked on the H\&E slides and 
the slides were submitted for molecular analysis. Genomic DNA samples were isolated from microdissected paraffin-embedded slides using a QIAamp DNA minikit (Qiagen, Germantown, MD, USA) according to the manufacturer's instructions. One milligram Chelex-100 resin (Bio-Rad, Hercules, CA, USA) was added, mixed to a slurry, and incubated at room temperature for $15 \mathrm{~min}$. After centrifugation at $10000 \mathrm{~g}$ for $5 \mathrm{~min}$, the supernatant was decanted for use. For PCR, we designed primer sets for exons 11,13 , and 17 of the KIT gene, as previously described. ${ }^{20}$ PCR was carried out in a total volume of $25 \mu \mathrm{l}$ containing 50-100 ng of genomic DNA and 0.25 acral lentiginous DNA polymerase (Bioline, London, UK). Mutations in these genes were identified by sequencing the PCR products on a $3730 \times 1$ DNA analyzer (Applied Biosystems, Carlsbad, CA, USA) in the Molecular Diagnostic Laboratory or the Nucleic Acid Core Facility. Control wild-type KIT sequences were used for comparison to evaluate for mutation.

\section{Statistical Analysis}

The statistical association between immunohistochemical expression of KIT, KIT genotype, and diagnoses was investigated using Fisher's exact test, Kruskal-Wallis test, and the Spearman's rank correlation when the ordering of both factors was important. The level of significance was set at $5 \%$.

\section{Results}

\section{Patient and Tumor Characteristics}

Table 1 summarizes patients' clinical characteristics and anatomical location of the analyzed tumors. Our series mainly included cases of acral lentiginous/ mucosal (primary and metastatic) melanoma $(n=141)$. A few cases corresponding to other types of melanoma $(n=23)$, as well as metastatic melanomas from unknown primaries were also included $(n=9)$.

Overall, patients presenting with acral lentiginous melanoma (primary and metastatic) showed a slight male predominance (39 cases, 56\%). There was a wide age range of presentation (24-87 years). The most common anatomical primary site was the foot. Only two cases arose on the upper extremities.

Mucosal melanoma patients were predominantly females ( 54 cases, $76 \%$ ). The age of presentation ranged widely from 26 to 85 years. The anorectal region was the most frequent primary site $(n=29)$, followed by the vulva/vagina/cervix area $(n=15)$. Head and neck primaries were also frequent $(n=14)$. A few urethral primary melanomas were also observed $(n=3)$.

Lymph nodes were the sites most frequently involved by metastatic tumors in our series $(n=27)$. Skin metastases (many of them representing in-transit metastases) were also present $(n=14)$. Mutational and immunohistochemical analyses were also performed on some metastatic melanomas

Table 1 Patient demographics and clinical characteristics of primary and metastatic melanoma cases

\begin{tabular}{|c|c|c|c|c|c|}
\hline \multirow[t]{2}{*}{ Diagnosis } & \multirow{2}{*}{$\begin{array}{c}\text { No. of } \\
\text { cases }(\%)\end{array}$} & \multicolumn{2}{|c|}{$\operatorname{Sex}(\%)$} & \multirow{2}{*}{$\begin{array}{l}\text { Mean age, } \\
\text { years (range) }\end{array}$} & \multirow[t]{2}{*}{ Anatomical site (n, \%) } \\
\hline & & $M$ & $F$ & & \\
\hline $\begin{array}{l}\text { Acral lentiginous, } \\
\text { primary }\end{array}$ & $46(27)$ & $28(61)$ & $18(39)$ & $60(24-87)$ & $\begin{array}{l}\text { Right foot NOS }(21,46) \text {, Left foot NOS }(12 \text {, } \\
26) \text {, Left toe }(4,9) \text {, Right toe }(3,7) \text {, Right heel } \\
(2,4) \text {, Left heel }(2,4) \text {, Right hand }(1,2) \text {, Left } \\
\text { finger }(1,2)\end{array}$ \\
\hline $\begin{array}{l}\text { Acral lentiginous, } \\
\text { metastatic }\end{array}$ & $24(14)$ & $11(46)$ & $13(54)$ & $68(43-83)$ & $\begin{array}{l}\text { Lymph node, inguinal }(13,54) \text {, skin }(10,42) \text {, } \\
\text { lung }(1,4)\end{array}$ \\
\hline Mucosal, primary & $61(35)$ & $14(23)$ & $47(77)$ & $62(26-85)$ & $\begin{array}{l}\text { Anus/rectum }(29,47) \text {, vulva }(11,18) \text {, } \\
\text { sinonasal }(9,15) \text {, vagina/cervix }(4,7) \text {, } \\
\text { conjunctiva }(4,7) \text {, urethra }(3,5) \text {, palate }(1,1)\end{array}$ \\
\hline Mucosal, metastatic & $10(6)$ & $3(30)$ & $7(70)$ & $60(49-71)$ & $\begin{array}{l}\text { Lymph nodes: inguinal }(5,50) \text {, neck }(3,30) \text {; } \\
\text { Soft tissue: neck }(1,10) \text {, perineum }(1,10)\end{array}$ \\
\hline Nodular, primary & $3(2)$ & $2(67)$ & $1(33)$ & $69(63-83)$ & Leg $(2,67)$, neck $(1,33)$ \\
\hline Nodular, metastatic & 2 (1) & $2(100)$ & $0(0)$ & $35(20-50)$ & Skin $(2,100)$ \\
\hline LMM, primary & $2(1)$ & $1(50)$ & $1(50)$ & $65(51-78)$ & Face $(1,50)$, scalp $(1,50)$ \\
\hline LMM, metastatic & $2(1)$ & $1(50)$ & $1(50)$ & $78(77-78)$ & Skin $(1,50)$, lung $(1,50)$ \\
\hline SS, metastatic & $3(2)$ & $2(67)$ & $1(33)$ & $63(53-80)$ & $\begin{array}{l}\text { Soft tissue: arm }(1,33) \text {, chest }(1,33) \text {, oral } \\
\text { cavity }(1,33)\end{array}$ \\
\hline Uveal, metastatic & $2(1)$ & $0(0)$ & $2(100)$ & $64(55-72)$ & Colon $(1,50)$, soft tissue: thigh $(1,50)$ \\
\hline Soft tissue & $1(0.5)$ & $1(100)$ & $0(0)$ & 77 & Peri-rectal $(1,100)$ \\
\hline $\begin{array}{l}\text { Unclassified, } \\
\text { metastatic }\end{array}$ & $8(4.5)$ & $5(63)$ & $3(37)$ & $60(40-80)$ & $\begin{array}{l}\text { Lymph nodes: neck }(2,25) \text {, axillary }(2,25) \text {, } \\
\text { inguinal }(2,25) \text {; skin }(1,17) \text {, lung }(1,17)\end{array}$ \\
\hline $\begin{array}{l}\text { Metastatic, unknown } \\
\text { primary }\end{array}$ & $9(5)$ & $4(44)$ & $5(56)$ & $51(30-65)$ & $\begin{array}{l}\text { Stomach }(2,22) \text {, small bowel }(2,22) \text {, } \\
\text { gallbladder }(1,11) \text {, soft tissue, abdomen }(1, \\
11) \text {, breast }(1,11) \text {, ovary }(1,11) \text {, brain }(1,11)\end{array}$ \\
\hline
\end{tabular}


Table 2 Immunohistochemical expression of KIT (percentage of positive cells) in primary and metastatic melanoma

\begin{tabular}{|c|c|c|c|c|c|c|}
\hline & \multicolumn{5}{|c|}{ Immunohistochemical expression of KIT (\%) } & \multirow[t]{2}{*}{$\mathrm{P}$-value } \\
\hline & 0 & $<5$ & $5-50$ & $51-95$ & $95+$ & \\
\hline \multicolumn{7}{|c|}{ Immunohistochemical expression of KIT (intensity) } \\
\hline 0 & $16(100 \%)$ & $0(0 \%)$ & $0(0 \%)$ & $0(0 \%)$ & $0(0 \%)$ & $<0.0001^{\mathrm{a}}$ \\
\hline $1+$ & $0(0 \%)$ & $12(75 \%)$ & $27(63 \%)$ & $8(17 \%)$ & $0(0 \%)$ & \\
\hline $2+$ & $0(0 \%)$ & $2(13 \%)$ & $12(28 \%)$ & $23(48 \%)$ & $6(25 \%)$ & \\
\hline $3+$ & $0(0 \%)$ & $2(13 \%)$ & $4(9 \%)$ & $17(35 \%)$ & $18(75 \%)$ & \\
\hline \multicolumn{7}{|l|}{ Diagnosis } \\
\hline ALM, metastatic & $5(31 \%)$ & $3(19 \%)$ & $7(16 \%)$ & $6(13 \%)$ & $3(13 \%)$ & $0.81^{\mathrm{b}}$ \\
\hline ALM, primary & $3(19 \%)$ & $3(19 \%)$ & $9(21 \%)$ & $14(29 \%)$ & $7(29 \%)$ & \\
\hline MuM, metastatic & $1(6 \%)$ & $0(0 \%)$ & $4(9 \%)$ & $3(6 \%)$ & $2(8 \%)$ & \\
\hline MuM, primary & $3(19 \%)$ & $5(31 \%)$ & $18(42 \%)$ & $15(31 \%)$ & $10(42 \%)$ & \\
\hline Non-ALM ${ }^{\mathrm{c}}$, metastatic & $4(25 \%)$ & $3(19 \%)$ & $4(25 \%)$ & $8(17 \%)$ & $2(8 \%)$ & \\
\hline Non-ALM ${ }^{\mathrm{c}}$, primary & $0(0 \%)$ & $2(13 \%)$ & $1(2 \%)$ & $2(4 \%)$ & $0(0 \%)$ & \\
\hline
\end{tabular}

ALM: acral lentiginous melanoma; MuM: mucosal melanoma.

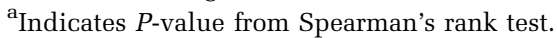

${ }^{\mathrm{b}} P$-value from Kruskal-Wallis test.

${ }^{\mathrm{C}}$ Includes other types of primary and metastatic melanoma and metastases from unknown origin.

from unknown primaries $(n=9)$, because of possible therapeutic implications. Sites for metastatic tumors from undiscovered primaries included gastrointestinal tract, soft tissue, ovary, and brain.

\section{Immunohistochemical Expression of KIT}

Immunohistochemical analysis for KIT was available in 121 out of 141 acral lentiginous/mucosal (primary and metastatic) melanomas and in 26 of the 32 remaining cases ('non-acral lentiginous/mucosal melanomas'). Overall, 32 cases $(22 \%)$ showed 0 to less than $5 \%$ of positive cells, $43(29 \%)$ cases $5-$ $50 \%, 48(33 \%)$ cases $51-95 \%$, and $24(16 \%)$ cases showed greater than $95 \%$ of cells expressing KIT. A high percentage of acral lentiginous/mucosal melanomas, both primary and metastatic, showed at least $5 \%$ of KIT-positive cells (98 of 121 cases, $81 \%$ ). The two uveal melanoma cases showed $>95 \%$ of tumor cells positive for KIT, with a moderate $(2+)$ intensity. Although, as a whole, the group of 'nonacral lentiginous/mucosal melanomas', primary and metastatic, displayed an apparently lower level of KIT expression (17 out of $26,65 \%$ ) compared with the acral lentiginous/mucosal melanomas, the small number of 'non- acral lentiginous/mucosal melanoma' cases considered for this study prevents conclusions in regards to level of expression of KIT by melanomas other than acral lentiginous/ mucosal type and may explain the lack of any statistical significant difference between the types of melanoma when compared by the level of KIT expression (Table 2). Similarly, intensity of immunohistochemical staining for KIT did not correlate with type of melanoma $(P=0.86$, data not shown). Intensity of staining and percentage of positive cells were positively correlated $(P<0.0001$, Table 2$)$.
Cytoplasmic staining with membranous accentuation was noted in most of the cases (116 cases, Figure 1).

Acral lentiginous and mucosal primary melanomas showed a comparable level of KIT immunohistochemical expression when cases showing at least $5 \%$ of positive cells were considered (30 out of 36 primary acral lentiginous and 43 out of 51 primary mucosal melanomas; 83 and $84 \%$, respectively). When a cutoff value of more than $50 \%$ of positive cells was considered, primary acral lentiginous and mucosal melanomas showed a slightly higher percentage of positive cases compared with the other groups (primary acral lentiginous, 58\%; primary mucosal, $61 \%$; metastatic acral lentiginous, $38 \%$; metastatic mucosal, $50 \%$; primary non-acral lentiginous/mucosal, $40 \%$; metastatic non-acral lentiginous/mucosal, $48 \%$ ).

Though cases are too few for statistical analysis, metastatic acral lentiginous melanomas seemed to be less likely to be KIT-positive than metastatic mucosal melanomas (16 out of 24 cases, $67 \%$ vs 9 out of 10 cases, $90 \%$, respectively).

A component of melanoma in situ was available for evaluation in 30 cases ( 21 acral lentiginous and nine mucosal melanomas). Almost all the in situ melanoma areas showed a $>95 \%$, strong $(3+)$ KIT immunolabeling (29 cases, 97\%, data not shown; Figure 2). The only case of melanoma in situ displaying moderate KIT expression (1 case, 3\%)scored as 51-95\%, moderate $(2+)$-corresponded to a conjunctival melanoma with a small invasive component.

In four patients (three mucosal and one acral lentiginous melanomas) both primary and metastatic tumors were available for immunohistochemical analysis. Molecular testing was performed on both primary and metastatic tumors as well. 

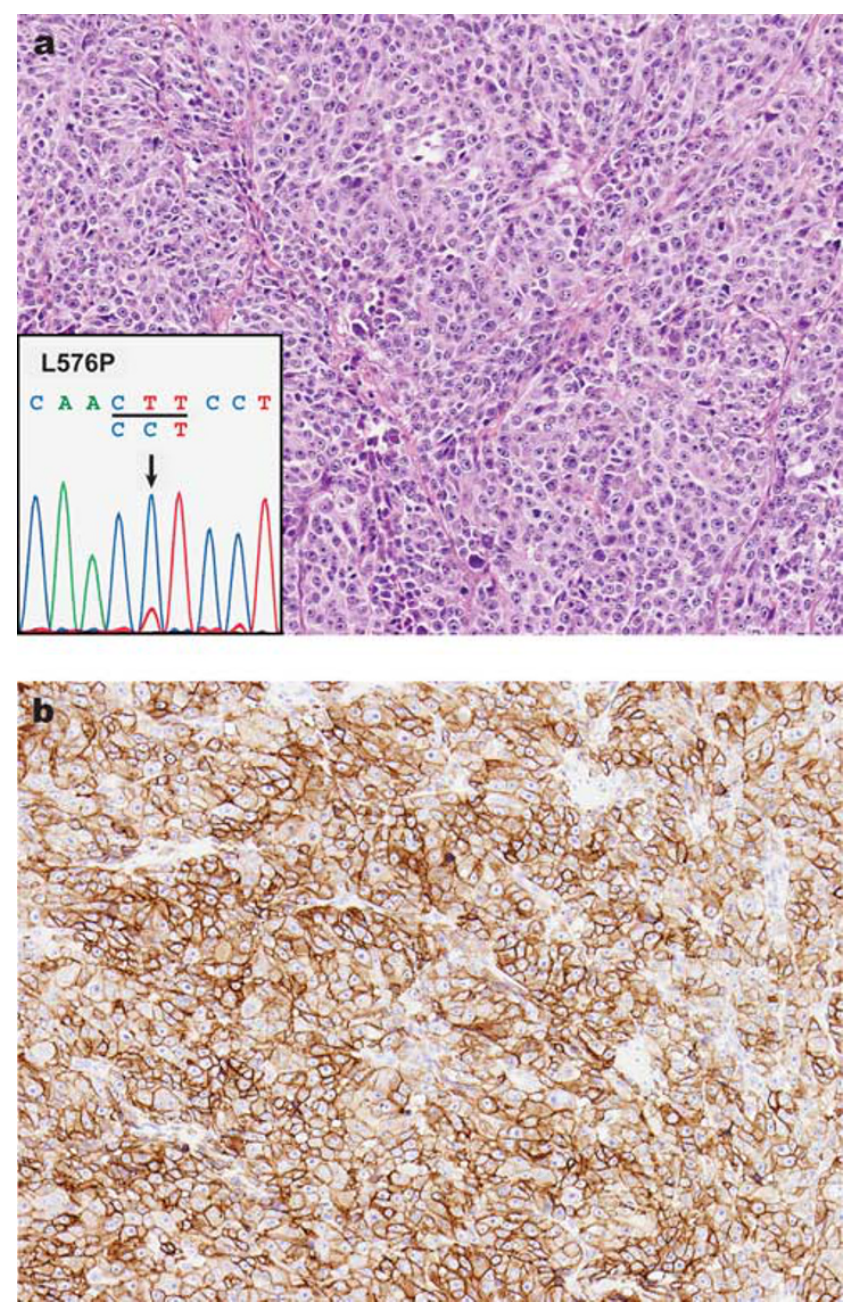

Figure 1 Strong and diffuse cytoplasmic expression of KIT by immunohistochemistry in a mucosal melanoma case harboring a L576P mutation in KIT (case no. 162). Notice the cell membrane reinforcement (a, H\&E, $\times 20$; b, immunohistochemical stain, $\times 20$; inset, sequence analysis of exon 11 of the KIT gene showing a point mutation in codon 576, resulting in the substitution of leucine by proline).

Immunohistochemical detection of KIT was comparable in the primary and the metastatic tumors in all four cases with each showing strong and diffuse expression of KIT. In two of the cases (one mucosal and one acral lentiginous melanoma), mutations of the KIT gene were found (Figure 3). The same molecular abnormalities were detected in these primary tumors and their cognate metastases (Table 3, Figure 3).

\section{Molecular Testing for KIT}

Molecular assay for KIT mutation was performed on 135 cases $(78 \%)$. In 15 of these cases (11\%), mutation status could not be determined because of low quality or insufficient DNA. From the cases in which amplifiable DNA was obtained $(n=120)$, 14 were found to harbor a specific, activating
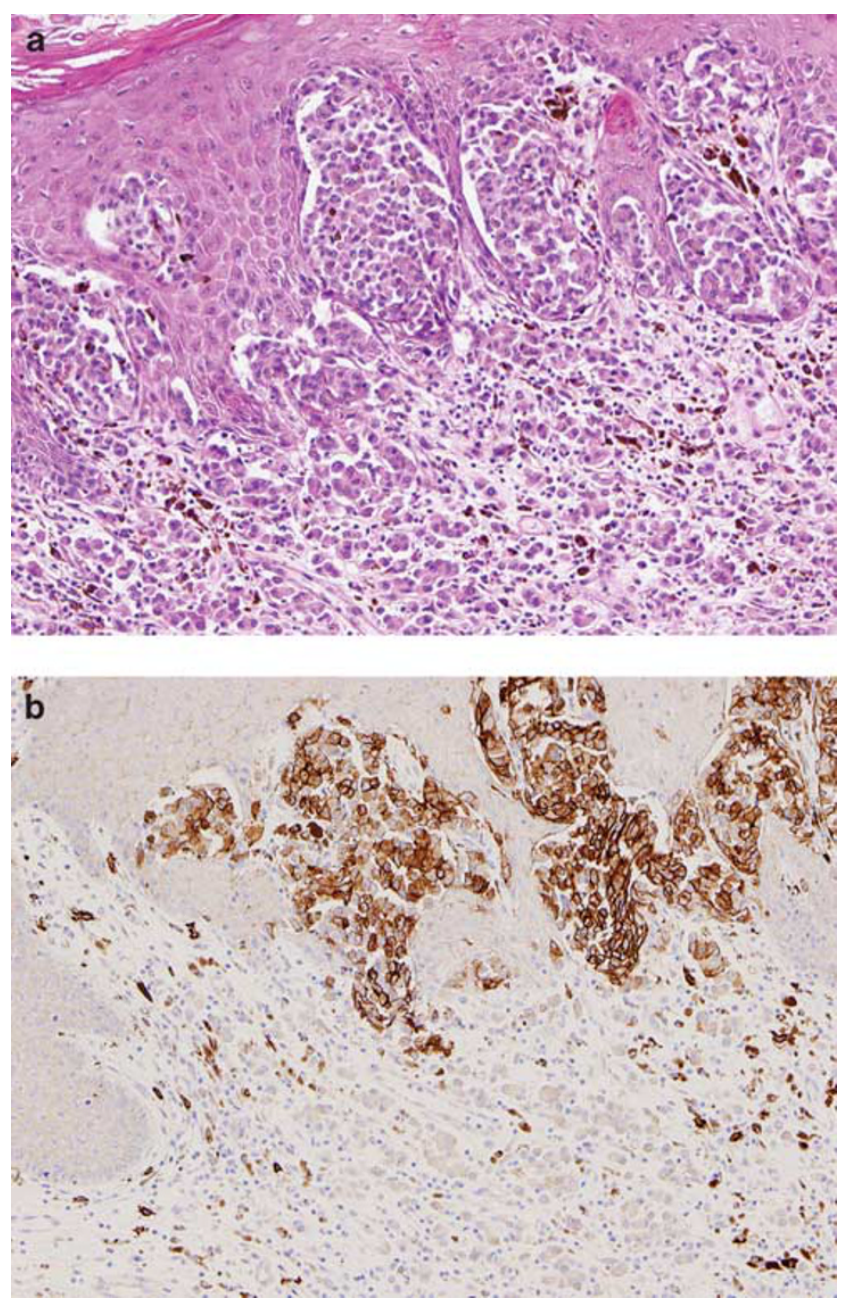

Figure 2 A case of acral-lentiginous melanoma showing strong and diffuse expression of KIT in the in situ component but absence of immunohistochemical staining in the invasive component. This case was found to be negative for mutations in KIT. $(\mathbf{a}, \times \mathrm{H} \& \mathrm{E}, 10 ; \mathbf{b}$, immunohistochemical stain, $\times 10)$.

KIT mutation (12\%). All the mutation-positive cases were acral lentiginous/mucosal melanoma (primary or metastatic). The overall frequency of KIT gene mutation in the acral lentiginous/ mucosal melanoma group was $15 \%$ (14 out of 91 informative cases). From these, five acral lentiginous melanoma cases $(13 \%, 5$ out of 39 informative cases) and nine mucosal melanomas carried a gene mutation for KIT $(17 \%, 9$ out of 52 informative cases). No KIT gene mutation was detected in any of the 'non-acral lentiginous/ mucosal melanomas' tested, including the two uveal melanomas.

Mutations were mapped in exons 11 (nine cases, 64\%), 13 (three cases, 21\%), and 17 (three cases, $21 \%)$. One case was found to harbor mutations in both exons 13 and 17 (Table 4). The most frequent mutation detected was L576P in exon 11 (four cases, $29 \%$, Figure 1); it constituted almost a half of the 

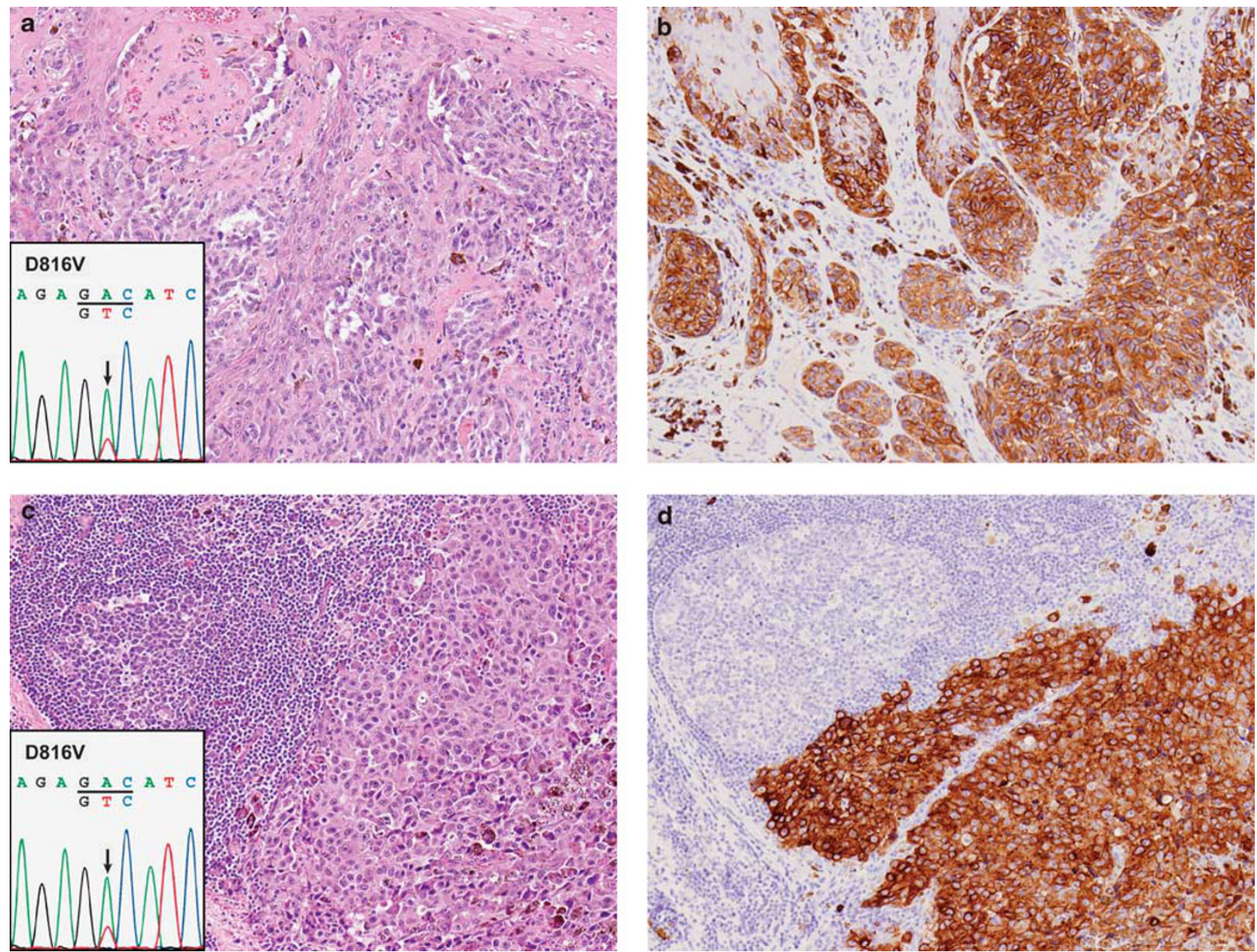

Figure 3 Mucosal melanoma (case no. 20), positive for the D816V KIT mutation. The primary tumor (a and b) shows a strong diffuse immunohistochemical expression of KIT in both in situ and invasive components. A lymph node metastasis (c and d) is also diffusely positive for KIT. (a, c, H\&E, $\times 10$; b, d, immunohistochemical stain, $\times 10$; inset, sequence analysis of exon 17 of the KIT gene showing a mutation in codon 816 which resulted in the substitution of aspartic acid by valine).

Table 3 Immunohistochemical and mutational status of KIT in cases in which primary and metastatic melanoma tumors from the same patient were available for study

\begin{tabular}{|c|c|c|c|c|}
\hline $\begin{array}{l}\text { Case } \\
\text { No. }\end{array}$ & Diagnosis & $\begin{array}{l}\text { KIT IHC, primary } \\
\text { tumor (site) }\end{array}$ & KIT IHC, metastasis (site) & Mutational analysis \\
\hline 9 & Mucosal melanoma & 51-95\%, 1+ (anus/rectum) & >95\%, 3+ (inguinal lymph node) & Not amplifiable DNA (both) \\
\hline 17 & Mucosal melanoma & $51-95 \%, 3+$ (urethra) & $51-95 \%, 2+$ (skin) & Not amplifiable DNA (both) \\
\hline 20 & Mucosal melanoma & $>95 \%, 3+$ (vagina) & $51-95 \%, 2+$ (inguinal lymph node) & $\begin{array}{l}\text { KIT mutation, exon 17, D816V } \\
\text { (both) }\end{array}$ \\
\hline 60 & $\begin{array}{l}\text { Acral lentiginous } \\
\text { melanoma }\end{array}$ & $51-95 \%, 2+$ (left toe) & $>95 \%, 3+$ (skin) & $\begin{array}{l}\text { KIT mutation, exon } 11 \text {, V559A } \\
\text { (both) }\end{array}$ \\
\hline
\end{tabular}

mutations found in exon 11 (four out of nine cases, $44 \%)$. Two cases displaying the L576P mutation in exon 11 were mucosal melanomas from the anorectal region. The other two cases were an acral lentiginous melanoma from the foot and a vulvar melanoma. Mutations in exons 11, 13, and 17 were detected in both acral lentiginous and mucosal subgroups. Cases with positive KIT-activating mutation represented examples from different ana- tomic sites (foot, toe, anus/rectum, vulva, vagina, conjunctiva, and palate).

\section{Correlation Between Immunohistochemistry and Molecular Testing for KIT}

Table 5 shows the comparisons made by mutation status, immunohistochemical analysis, and melanoma type. There was evidence that the immunohisto- 
Table 4 Acral lentiginous/ mucosal melanoma cases positive for KIT mutation

\begin{tabular}{|c|c|c|c|c|}
\hline Case & Diagnosis & Kit mutation & KIT IHC (\%) & KIT IHC (intensity) \\
\hline 28 & AL, primary, left foot & Exon 11, L576P & $5-50$ & $1+$ \\
\hline 46 & $\mathrm{AL}$, primary, left foot & Exon 17, D816V & $51-95$ & $3+$ \\
\hline 82 & AL, primary, left foot & Exon 11,566 to 572 del & $5-50$ & $1+$ \\
\hline 52 & AL, met, inguinal LN (left foot primary) & Exon 13 , N655K & $>95$ & $3+$ \\
\hline 60 & $\mathrm{AL}$, met, skin (left toe primary) ${ }^{1}$ & Exon $11, V_{559}{ }^{a}$ & $51-95$ & $2+$ \\
\hline 6 & Muc, primary, anus/rectum ${ }^{\mathrm{a}}$ & Exon 11, V560D & $51-95$ & $3+$ \\
\hline 10 & Muc, primary, vulva & Exon 11, L576P & $51-95$ & $3+$ \\
\hline 20 & Muc, primary, vagina ${ }^{a}$ & Exon $17, \mathrm{D}^{2} 16 \mathrm{~V}^{\mathrm{a}}$ & $51-95$ & $2+$ \\
\hline 38 & Muc, primary, conjunctiva & Exon 11, V559G & $>95$ & $2+$ \\
\hline 88 & Muc, primary, palate & Exon 11,566 to 574 del & $51-95$ & $2+$ \\
\hline 120 & Muc, primary, anus/rectum & Exon 11, L576P & $>95$ & $3+$ \\
\hline 127 & Muc, primary, vulva & Exon $13(\mathrm{~K} 642 \mathrm{E})$ and $17(\mathrm{~N} 822 \mathrm{I})$ & $>95$ & $2+$ \\
\hline 159 & Muc, primary, vulva & Exon 13, K642E & $>95$ & $3+$ \\
\hline 162 & Muc, primary, anus/rectum & Exon 11, L576P & $>95$ & $3+$ \\
\hline
\end{tabular}

AL: acral lentiginous; Muc: mucosal; met: metastasis.

${ }^{\mathrm{a}}$ Mutation analysis performed on both primary and metastatic tumors showed the same genetic defect.

Table 5 Correlation between immunohistochemical expression of KIT, KIT mutation status, and type of melanoma

\begin{tabular}{|c|c|c|c|}
\hline & \multicolumn{2}{|c|}{ Mutation status } & \multirow[t]{2}{*}{ P-value } \\
\hline & Negative & Positive & \\
\hline \multicolumn{4}{|c|}{ Immunohistochemical expression of KIT (\%) } \\
\hline 0 & $12(12)$ & $0(0)$ & $0.007^{\mathrm{a}}$ \\
\hline$<5$ & $14(14)$ & $0(0)$ & \\
\hline $5-50$ & $30(31)$ & $2(14)$ & \\
\hline $51-95$ & $31(32)$ & $6(43)$ & \\
\hline $95+$ & $10(10)$ & $6(43)$ & \\
\hline \multicolumn{4}{|c|}{ Immunohistochemical expression of KIT (intensity) (\%) } \\
\hline 0 & $12(12)$ & $0(0)$ & $0.08^{\mathrm{b}}$ \\
\hline $1+$ & 36 (37) & $2(14)$ & \\
\hline $2+$ & $25(26)$ & $5(36)$ & \\
\hline $3+$ & $24(25)$ & $7(50)$ & \\
\hline \multicolumn{4}{|l|}{ Diagnosis } \\
\hline ALM met & $12(11)$ & $2(14)$ & $0.14^{\mathrm{b}}$ \\
\hline ALM primary & $22(21)$ & $3(21)$ & \\
\hline MucM met & $8(8)$ & $0(0)$ & \\
\hline MucM primary & 35 (33) & $9(64)$ & \\
\hline Melanoma, other, met & $24(23)$ & $0(0)$ & \\
\hline Melanoma, other, primary & $5(5)$ & $0(0)$ & \\
\hline
\end{tabular}

ALM: acral lentiginous melanoma; MucM: mucosal melanoma; met: metastasis.

${ }^{a}$ Indicates $P$-value from Kruskal-Wallis test.

${ }^{\mathrm{b}}$ Indicates $P$-value from Fisher's exact test.

chemical detection of KIT (expressed as percentage of positive cells) was associated with mutation status $(P=0.007)$. A trend toward association of staining intensity and mutation status was also observed $(P=0.08)$.

When tumors were classified as acral lentiginous, mucosal, and other (primary and metastatic), no definitive association between melanoma type and mutation status was found by statistical analysis $(P=0.14)$. However, there is an obvious association between the acral lentiginous/mucosal group (considered as a whole) and KIT mutation, as all the positive cases were acral lentiginous/mucosal melanomas, and none of the 'non-acral lentiginous/ mucosal' cases was found to carry any mutation.

None of the cases showing $<5 \%$ positive tumor cells was found to harbor KIT mutations (0 of 32 cases), indicating a negative predictive value of $100 \%$ in this study. Immunohistochemical expression of KIT was identified in more than $50 \%$ of the tumor cells in $12(86 \%)$ of the 14 cases shown to be positive for KIT mutation (Table 5). The two positive cases for KIT mutation in which KIT expression was scored as 5 to $50 \%$ showed actual percentages of 10 and $20 \%$.

In four of the 14 cases positive for mutation in the KIT gene (case nos. 28, 82, 127, and 159, Table 4), areas of melanoma in situ were available for immunohistochemical assessment. All the in situ tumors showed diffuse and strong positivity for KIT $(>95 \%, 3+)$. The corresponding invasive component (where microdissection for molecular analysis was performed) showed a variable expression of KIT, with two cases displaying $5-50 \%$ of positive cells with weak intensity $(1+)$ (case nos. 28 and 82) and two cases showing diffuse and strong immunolabeling for KIT (case nos. 127 and 159, Table 4).

\section{Discussion}

Controversy still exists about the expression of KIT protein in melanoma. Initial studies reported an either reduced or absent immunohistochemical expression of KIT in invasive ('vertical growth phase') melanomas and metastatic tumors ${ }^{5,6}$ along with lack of functional KIT and KIT mRNA in 'advanced nodular' melanoma cell lines. ${ }^{21,22}$ Uveal melanomas, although reported to express KIT, ${ }^{7}$ were found to be negative for KIT mutations. ${ }^{17}$ On the other hand, Shen et $a l^{23}$ reported that $96 \%$ of primary melanomas expressed KIT by immunohistochemistry whereas $55 \%$ of metastatic melanomas 
were found to be negative. Since then, overexpression of KIT has been reported in $53.7 \%$ of superficial spreading melanomas, ${ }^{24} 75 \%$ of primary anorectal melanomas, ${ }^{25}$ metastases from the so-called 'malignant blue nevus', ${ }^{26}$ and in more than $80 \%$ of metastatic melanomas. ${ }^{27}$

Our results are in keeping with those recent studies showing that, in at least some types of melanoma, significant expression of KIT is present and can be detected by immunohistochemistry. We used a highly sensitive detection method employing a rabbit polyclonal antibody, widely tested in previous trials. ${ }^{23,28}$ It has been reported that heatinduced antigen retrieval may surprisingly reduce staining intensity with this particular antibody. ${ }^{28}$ Although our staining protocol included heatinduced antigen retrieval, our high rate of positive cases with adequate controls speaks against a suboptimal detection of positive cells. It is plausible that the apparent discordance between the initial and recent reports of immunohistochemical detection of KIT in melanoma may be, at least in part, due to the utilization of melanoma cell lines in earlier studies. ${ }^{16}$

It appears that, in the acral lentiginous/mucosal type, KIT expression by melanoma cells can be as high as $84 \%$. It seems that acral lentiginous/mucosal melanomas express KIT in a higher proportion of cases compared with other 'non-acral lentiginous/ mucosal' melanomas (found to express KIT in roughly $65 \%$ of the cases), except for uveal melanomas.

In our hands, metastatic melanomas tended to retain KIT expression. Loss of the protein expression by metastases was evident, at some extent, only in the acral lentiginous melanoma subgroup (83 and $67 \%$ in primary and metastatic tumors, respectively) whereas the mucosal melanoma subgroup showed at least a comparable level of expression (84 and 90\%, respectively). Three cases of mucosal melanoma displayed similar high levels of expression of KIT in both primary tumor and corresponding metastasis. Two cases, a mucosal melanoma and an acral lentiginous melanoma, highly expressed KIT and harbored an activating mutation of the gene in both the primary and its metastatic tumor. The high level of KIT expression in invasive and metastatic melanomas found in our study may suggest that loss of expression of KIT by itself might not have a direct role in melanocyte transformation and tumor progression in acral lentiginous/mucosal melanomas. ${ }^{21}$ It has been postulated that lack of KIT overexpression in melanoma cell lines is most likely due to altered expression of transcription factors such as AP-2, which results in downregulation of $\mathrm{KIT}^{29,30}$

Most of our cases showed cytoplasmic and membranous pattern of immunohistochemical expression. It has been proposed that cytoplasmic immunoreactivity for KIT may be due to a nonmature protein rather than a proteolytically pro- cessed molecule, which would be the active, membranous receptor form. In any case, in all our cases the two cellular locations were detected and no association between immunohistochemical staining pattern and mutational status of KIT was noted.

Fifteen of 135 (11\%) cases analyzed for KIT mutations were non-informative because of low quality or insufficient DNA and possibly inhibition of PCR amplification by melanin pigment ${ }^{31}$ or other inhibiting substances. A binding resin, used to purify the DNA, improved the rate of amplification significantly. Supplementing the PCR with bovine serum albumin to sequester melanin can also be helpful. ${ }^{32}$

The first case of a KIT mutation-positive melanoma (an L576P activation mutation in exon 11) was published in 2004 by Went et $a l^{8}$ Since then, two cases of metastatic melanoma from unknown primary $^{9}$ and three primary anal melanomas ${ }^{18}$ have been reported to harbor the same mutation. In 2006, Curtin et $a l^{16}$ found mutations and/or copy number increases of KIT in $39 \%$ of mucosal, $36 \%$ of acral, and $28 \%$ of melanomas on chronically sun-damaged skin; specific KIT mutations were shown in three out of $28(11 \%)$ primary acral and 8 of $38(21 \%)$ primary mucosal melanomas. No metastatic melanomas were included in their case series. Mucosal melanomas from the oral cavity have been reported to demonstrate mutations in KIT in 4 out of 18 cases. ${ }^{33}$ In a recent study, Beadling et $a l^{17}$ reported 23 and $15.6 \%$ of acral lentiginous and mucosal melanomas to be positive for KIT mutations, respectively.

We found that $13 \%$ of acral lentiginous and $17 \%$ of mucosal primary and metastatic melanomas harbor an activating mutation in KIT. None of the few other types of melanomas analyzed showed KIT mutations, including four cases of primary and metastatic lentigo maligna melanoma. Our results expand the previously reported data because both primary and metastatic tumors were included in our analysis. We were able to show the same activating mutations in primary tumors and their metastases in three patients (Table 4).

KIT mutations in acral lentiginous/mucosal melanomas most frequently occurred in exon 11 (9 out of 14 cases, 64\%). The L576P substitution constituted $44 \%$ of the exon 11 mutations. This mutation affects the juxta-membrane domain of KIT, promoting dimerization of the protein and its constitutive activation. ${ }^{16}$ One of our cases was found to carry the V559A mutation in exon 11 in both primary and metastatic tumors. This substitution, as L576P, constitutes a target for imatinib mesylate therapy. ${ }^{16,17}$ One of our cases, a primary anorectal mucosal melanoma harboring the V560D mutation, was previously reported..$^{12}$ Other mutations in exon 11 , not previously found in melanomas, were the V559G substitution and deletions of codons 566572 and 566-574. ${ }^{34-36}$ Mutations found in exon 13 were K642E, which affects the first tyrosine kinase 
domain and has been reported in melanomas and GIST, ${ }^{16,37,38}$ and N655K, which has recently been identified in a case of GIST and found to be of gainof-function and imatinib mesylate sensitive. ${ }^{39}$ One case harboring the K642E mutation was identified to carry a second mutation, N822I in exon 17. Evidence exists that $\mathrm{K} 642 \mathrm{E}$ is a weakly activating mutation that requires additional genetic alterations to be fully oncogenic. ${ }^{16}$ Our finding may support this contention. Mutations detected in exon 17, which encodes the second tyrosine kinase catalytic domain of KIT, ${ }^{40}$ were D816V and N822I. D816V has been previously reported to occur in acute myeloid leukemia and mastocytosis ${ }^{41,42}$ but not in melanoma. The encoded KIT mutant protein has been found to be completely insensitive to imatinib mesylate. ${ }^{43}$ A mutation in the same site, $\mathrm{D} 816 \mathrm{H}$, has been identified in imatinib mesylate-resistant GIST, ${ }^{40}$ germ-cell tumors, ${ }^{44}$ and two cases of mucosal and chronic sun-induced damage melanomas. ${ }^{16}$ N822I has not been reported before. However, secondary mutations between aminoacids 820 and 823 (such as $\mathrm{N} 822 \mathrm{~K}$ and $\mathrm{N822H}$ ) are considered in GIST as a common mechanism underlying the development of acquired resistance of tumors to imatinib mesylate. ${ }^{40,45,46}$

Our study shows a significant correlation between percentage of KIT-positive cells by IHC and KIT mutation status in invasive acral lentiginous/mucosal melanomas (Table $5, P=0.007$ ). No cases with less than $10 \%$ positive cells for KIT were found to harbor KIT mutations in exons 11,13 or 17 . It seems, therefore, that immunohistochemical expression of KIT, recorded as percentage of positive cells in the invasive component, is a strong negative predictor for mutation status when a cutoff value of $10 \%$ is considered. Immunohistochemical expression of KIT by the in situ component of acral lentiginous and mucosal melanomas seems to be consistently diffuse and strong, regardless of the gene mutation status, and therefore, not useful in predicting mutation of KIT.

Seventy-one $(64 \%)$ of the cases negative for $K I T$ mutation showed $5 \%$ or more positive cells for KIT. Some mechanisms other than gene mutations, such as increased numbers of copies of the gene ${ }^{16,47}$ and co-overexpression of cyclin-dependent kinase 4 and $\mathrm{KIT}^{48}$ may account for this finding. Epigenetic factors, such as the surrounding stroma ${ }^{5}$ and autocrine or paracrine stimulation ${ }^{49}$ may also have a role in the overexpression of KIT in acral lentiginous/ mucosal melanomas.

Lack of clinical efficacy of imatinib mesylate has been reported in a series of patients with uveal melanomas overexpressing KIT but negative for KIT mutations. ${ }^{50}$ Similar findings in primary cutaneous and metastatic melanomas ${ }^{27,51,52}$ have led some to postulate that imatinib mesylate is effective only in tumors harboring mutations in KIT, similar to what has been reported in studies exploring the in vitro sensitivity of melanoma cells to the drug. ${ }^{18,19}$ The importance of amplification of the KIT locus in predicting response to therapy remains an open and important question. ${ }^{16,17}$ The in vivo effect of imatinib mesylate measured as reduction of immunohistochemical expression of protein tyrosine kinases after treatment has been reported. ${ }^{53}$ Complete and major clinical responses to imatinib mesylate have been reported in two cases of metastatic anorectal mucosal melanomas carrying the K642E mutation and a seven codon duplication in exon 11, both strongly expressing KIT by immunohistochemistry. ${ }^{13,15}$ We have reported a case of metastatic mucosal melanoma from the anus/rectum harboring the V560D mutation and diffusely immunoreactive for KIT that showed complete response for 5 months to sorafenib, another receptor tyrosine kinase inhibitor. ${ }^{12}$ However, a recent phase II clinical trial, carried out in our institution, found only one case (a patient with metastatic acral lentiginous melanoma) to dramatically improve with imatinib mesylate and to have a partial response for more than a year. ${ }^{11}$ No genomic mutation in KIT was found in the responder case but the immunohistochemical expression of KIT was diffuse and scored as the highest among the tissue samples analyzed. Thus, it seems reasonable to consider those tumors with diffuse immunolabeling for KIT as potential candidates for both mutational analysis and treatment trial with tyrosine kinase inhibitors.

In summary, our study represents, to our knowledge, the largest series of acral lentiginous/mucosal melanoma cases in which both immunohistochemical and KIT genotyping have been performed. Immunohistochemical expression of KIT in less than $10 \%$ of the cells of the invasive component of acral lentiginous/mucosal melanomas appears to be a strong negative predictor of KIT mutation and therefore can potentially be used to triage cases for additional KIT genotyping.

\section{Disclosure/conflict of interest}

The authors declare no conflict of interest.

\section{References}

1 Grichnik JM, Burch JA, Burchette J, et al. The SCF/KIT pathway plays a critical role in the control of normal human melanocyte homeostasis. J Invest Dermatol 1998;111:233-238.

2 Grichnik JM. Kit and melanocyte migration. J Invest Dermatol 2006;126:945-947.

3 Holden JA, Willmore-Payne C, Layfield LJ. Tyrosine kinase activating mutations in human malignancies: implications for diagnostic pathology. Exp Mol Pathol 2008;85:68-75.

4 Alexeev V, Yoon K. Distinctive role of the cKit receptor tyrosine kinase signaling in mammalian melanocytes. J Invest Dermatol 2006;126:1102-1110.

5 Natali PG, Nicotra MR, Winkler AB, et al. Progression of human cutaneous melanoma is associated with loss 
of expression of c-kit proto-oncogene receptor. Int J Cancer 1992;52:197-201.

6 Montone KT, van Belle P, Elenitsas R, et al. Protooncogene c-kit expression in malignant melanoma: protein loss with tumor progression. Mod Pathol 1997;10:939-944.

7 Guerriere-Kovach PM, Hunt EL, Patterson JW, et al. Primary melanoma of the skin and cutaneous melanomatous metastases: comparative histologic features and immunophenotypes. Am J Clin Pathol 2004;122: 70-77.

8 Went PT, Dirnhofer S, Bundi M, et al. Prevalence of KIT expression in human tumors. J Clin Oncol 2004; 22:4514-4522.

9 Willmore-Payne C, Holden JA, Tripp S, et al. Human malignant melanoma: detection of BRAF- and c-kitactivating mutations by high-resolution amplicon melting analysis. Hum Pathol 2005;36:486-493.

10 Fiorentini G, Rossi S, Lanzanova G, et al. Potential use of imatinib mesylate in ocular melanoma and liposarcoma expressing immunohistochemical c-KIT (CD117). Ann Oncol 2003;14:805.

11 Kim KB, Eton O, Davis DW, et al. Phase II trial of imatinib mesylate in patients with metastatic melanoma. Br J Cancer 2008;99:734-740.

12 Quintas-Cardama A, Lazar AJ, Woodman SE, et al. Complete response of stage IV anal mucosal melanoma expressing KIT Val560Asp to the multikinase inhibitor sorafenib. Nat Clin Pract Oncol 2008;5:737-740.

13 Hodi FS, Friedlander P, Corless CL, et al. Major response to imatinib mesylate in KIT-mutated melanoma. J Clin Oncol 2008;26:2046-2051.

14 Heinrich MC, Joensuu H, Demetri GD, et al. Phase II, open-label study evaluating the activity of imatinib in treating life-threatening malignancies known to be associated with imatinib-sensitive tyrosine kinases. Clin Cancer Res 2008;14:2717-2725.

15 Lutzky J, Bauer J, Bastian BC. Dose-dependent, complete response to imatinib of a metastatic mucosal melanoma with a K642E KIT mutation. Pigment Cell Melanoma Res 2008;21:492-493.

16 Curtin JA, Busam K, Pinkel D, et al. Somatic activation of KIT in distinct subtypes of melanoma. J Clin Oncol 2006;24:4340-4346.

17 Beadling C, Jacobson-Dunlop E, Hodi FS, et al. KIT gene mutations and copy number in melanoma subtypes. Clin Cancer Res 2008;14:6821-6828.

18 Antonescu CR, Busam KJ, Francone TD, et al. L576P KIT mutation in anal melanomas correlates with KIT protein expression and is sensitive to specific kinase inhibition. Int J Cancer 2007;121: 257-264.

19 Jiang X, Zhou J, Yuen NK, et al. Imatinib targeting of KIT-mutant oncoprotein in melanoma. Clin Cancer Res 2008;14:7726-7732.

20 Chen LL, Trent JC, Wu EF, et al. A missense mutation in KIT kinase domain 1 correlates with imatinib resistance in gastrointestinal stromal tumors. Cancer Res 2004;64:5913-5919.

21 Lassam N, Bickford S. Loss of c-kit expression in cultured melanoma cells. Oncogene 1992;7:51-56.

22 Funasaka Y, Boulton T, Cobb M, et al. c-Kit-kinase induces a cascade of protein tyrosine phosphorylation in normal human melanocytes in response to mast cell growth factor and stimulates mitogen-activated protein kinase but is down-regulated in melanomas. Mol Biol Cell 1992;3:197-209.
23 Shen SS, Zhang PS, Eton O, et al. Analysis of protein tyrosine kinase expression in melanocytic lesions by tissue array. J Cutan Pathol 2003;30:539-547.

24 Potti A, Hille RC, Koch M. Immunohistochemical determination of HER-2/neu overexpression in malignant melanoma reveals no prognostic value, while c-Kit (CD117) overexpression exhibits potential therapeutic implications. J Carcinog 2003;2:8.

25 Chute DJ, Cousar JB, Mills SE. Anorectal malignant melanoma: morphologic and immunohistochemical features. Am J Clin Pathol 2006;126:93-100.

26 Zyrek-Betts J, Micale M, Lineen A, et al. Malignant blue nevus with lymph node metastases. J Cutan Pathol 2008;35:651-657.

27 Alexis JB, Martinez AE, Lutzky J. An immunohistochemical evaluation of c-kit (CD-117) expression in malignant melanoma, and results of imatinib mesylate (Gleevec) therapy in three patients. Melanoma Res 2005;15:283-285.

28 Hornick JL, Fletcher CD. Immunohistochemical staining for KIT (CD117) in soft tissue sarcomas is very limited in distribution. Am J Clin Pathol 2002; 117:188-193.

29 Zakut R, Perlis R, Eliyahu S, et al. KIT ligand (mast cell growth factor) inhibits the growth of KIT-expressing melanoma cells. Oncogene 1993;8: 2221-2229.

30 Huang S, Jean D, Luca M, et al. Loss of AP-2 results in downregulation of c-KIT and enhancement of melanoma tumorigenicity and metastasis. EMBO J 1998;17: 4358-4369.

31 Eckhart L, Bach J, Ban J, et al. Melanin binds reversibly to thermostable DNA polymerase and inhibits its activity. Biochem Biophys Res Commun 2000;271: 726-730.

32 Giambernardi TA, Rodeck U, Klebe RJ. Bovine serum albumin reverses inhibition of RT-PCR by melanin. Biotechniques 1998;25:564-566.

33 Rivera RS, Nagatsuka H, Gunduz M, et al. C-kit protein expression correlated with activating mutations in KIT gene in oral mucosal melanoma. Virchows Arch 2008;452:27-32.

34 Haller F, Gunawan B, von Heydebreck A, et al. Prognostic role of E2F1 and members of the CDKN2A network in gastrointestinal stromal tumors. Clin Cancer Res 2005;11:6589-6597.

35 Lasota J, Jasinski M, Sarlomo-Rikala M, et al. Mutations in exon 11 of c-Kit occur preferentially in malignant $v s$ benign gastrointestinal stromal tumors and do not occur in leiomyomas or leiomyosarcomas. Am J Pathol 1999;154:53-60.

36 Debiec-Rychter M, Sciot R, Le Cesne A, et al. KIT mutations and dose selection for imatinib in patients with advanced gastrointestinal stromal tumours. Eur J Cancer 2006;42:1093-1103.

37 McAuliffe JC, Wang WL, Pavan GM, et al. Unlucky number 13? Differential effects of KIT exon 13 mutation in gastrointestinal stromal tumors. Mol Oncol 2008;2:161-163.

38 Lasota J, Corless CL, Heinrich MC, et al. Clinicopathologic profile of gastrointestinal stromal tumors (GISTs) with primary KIT exon 13 or exon 17 mutations: a multicenter study on 54 cases. Mod Pathol 2008; 21:476-484.

39 Kinoshita K, Hirota S, Isozaki K, et al. Characterization of tyrosine kinase I domain c-kit gene mutation Asn655Lys newly found in primary jejunal gastro- 
intestinal stromal tumor. Am J Gastroenterol 2007; 102:1134-1136.

40 Desai J, Shankar S, Heinrich MC, et al. Clonal evolution of resistance to imatinib in patients with metastatic gastrointestinal stromal tumors. Clin Cancer Res 2007;13:5398-5405.

41 Beghini A, Cairoli R, Morra E, et al. In vivo differentiation of mast cells from acute myeloid leukemia blasts carrying a novel activating ligand-independent C-kit mutation. Blood Cells Mol Dis 1998;24: 262-270.

42 Verzijl A, Heide R, Oranje AP, et al. C-kit Asp-816-Val mutation analysis in patients with mastocytosis. Dermatology 2007;214:15-20.

43 Frost MJ, Ferrao PT, Hughes TP, et al. Juxtamembrane mutant V560GKit is more sensitive to Imatinib (STI571) compared with wild-type c-kit whereas the kinase domain mutant D816VKit is resistant. Mol Cancer Ther 2002;1:1115-1124.

44 Tian Q, Frierson Jr HF, Krystal GW, et al. Activating c-kit gene mutations in human germ cell tumors. Am J Pathol 1999;154:1643-1647.

45 Debiec-Rychter M, Cools J, Dumez H, et al. Mechanisms of resistance to imatinib mesylate in gastrointestinal stromal tumors and activity of the PKC412 inhibitor against imatinib-resistant mutants. Gastroenterology 2005;128:270-279.

46 Antonescu CR, Besmer P, Guo T, et al. Acquired resistance to imatinib in gastrointestinal stromal tumor occurs through secondary gene mutation. Clin Cancer Res 2005;11:4182-4190.

47 Bastian BC, LeBoit PE, Hamm H, et al. Chromosomal gains and losses in primary cutaneous melanomas detected by comparative genomic hybridization. Cancer Res 1998;58:2170-2175.

48 Smalley KS, Contractor R, Nguyen TK, et al. Identification of a novel subgroup of melanomas with KIT/ cyclin-dependent kinase-4 overexpression. Cancer Res 2008;68:5743-5752.

49 Lefevre G, Glotin AL, Calipel A, et al. Roles of stem cell factor/c-Kit and effects of Glivec/STI571 in human uveal melanoma cell tumorigenesis. J Biol Chem 2004; 279:31769-31779.

50 Hofmann UB, Kauczok-Vetter CS, Houben R, et al. Overexpression of the KIT/SCF in uveal melanoma does not translate into clinical efficacy of imatinib mesylate. Clin Cancer Res 2009;15:324-329.

51 Wyman K, Atkins MB, Prieto V, et al. Multicenter Phase II trial of high-dose imatinib mesylate in metastatic melanoma: significant toxicity with no clinical efficacy. Cancer 2006;106:2005-2011.

52 Ugurel S, Hildenbrand R, Zimpfer A, et al. Lack of clinical efficacy of imatinib in metastatic melanoma. Br J Cancer 2005;92:1398-1405.

53 Ivan D, Niveiro M, Diwan AH, et al. Analysis of protein tyrosine kinases expression in the melanoma metastases of patients treated with Imatinib Mesylate (STI571, Gleevec). J Cutan Pathol 2006;33:280-285. 